1996;60:297-300). Apnea occurred in 20 of 47 clinical seizures and 10 of 17 patients. It was generally central, but obstructive apnea occurred in 3 . Oxyhemoglobin saturation dropped to $<85 \%$, and tachycardia was common. Bradycardia/sinus arrest was documented with a change in respiration in 4 . Similar mechanisms involving cardiorespiratory reflexes are suggested in relation to cases of sudden death in epilepsy.

\title{
DIABETES INSIPIDUS WITH HYPOXIC ENCEPHALOPATHY
}

Neurogenic diabetes insipidus (DI) due to hypoxic encephalopathy is reported in 6 cases and 28 pediatric cases in the literature are reviewed from the Mackay Memorial Hospital, Taipei, Taiwan, ROC. The ages ranged from infancy to 18 years (mean 7 years), and the interval from the hypoxia to the onset of DI was a mean of 4 days. Two neonates survived but had developmental delay. The remaining 26 patients died. DI is a sign of severe brain damage, and this potential complication should be suspected in patients with polyuria and dehydration following hypoxic encephalopathy. (Lee Y-J, Huang F-Y et al. Neurogenic diabetes insipidus in children with hypoxic encephalopathy: six new cases and a review of the literature. Eur I Pediatr March 1996;155:245-248). (Respond: Dr Fu-Yuan Huang, Department of Paediatrics, Mackay Memorial Hospital, Taipei, 10449 Taiwan, ROC).

COMMENT. Neurogenic diabetes insipidus may be caused by head trauma, meningitis, encephalitis, a complication of brain surgery, in addition to hypoxia. Fluid intake and output, plasma sodium, and urine specific gravity should be monitored in children following hypoxic/ischemic insults or in other critically ill brain injured patients.

\section{INFECTIOUS DISEASES}

\section{NEUROLOGIC SIGNS OF LYME DISEASE}

Clinical manifestations of Lyme disease (LD) in 97 seropositive children were reviewed at the Children's Hospital of Philadelphia, with particular attention to neurologic symptoms and signs. Of 69 children with LD, 22 (32\%) had new neurologic abnormalities, mainly facial palsy and aseptic meningitis. Only $27 \%$ of children with neurologic abnormalities due to LD had a history of erythema migrans or arthritis. Seropositivity for LD and neurologic symptoms usually indicates an active neuroborreliosis. (Bingham PM et al. Neurologic manifestations in children with Lyme disease. Pediatrics Dec 1995;96:10531056). (Respond: Dr Peter M Bingham, Division of Neurology, Children's Hospital of Philadelphia, 34th Street and Civic Center Blvd, Philadelphia, PA 19104).

COMMENT. Definite evidence for neuroborreliosis requires characteristic neurologic abnormalities and either erythema migrans, arthritis or heart involvement, positive spinal fluid serology, or seroconversion. Peripheral neuropathy is infrequent in children compared to adults. Facial palsy and aseptic meningitis are the most frequent neurologic manifestations of $\mathrm{LD}$ in children. In the approaching summer months, children presenting with these disorders should be checked for possible Lyme disease. 